\title{
Instrumentation for Time-of-Flight Positron Emission Tomography
}

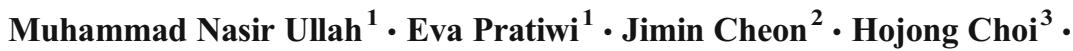 \\ Jung Yeol Yeom ${ }^{1,4}$
}

Received: 7 October 2015 /Revised: 8 December 2015 /Accepted: 25 January 2016/Published online: 22 February 2016

(C) Korean Society of Nuclear Medicine 2016

\begin{abstract}
Positron emission tomography (PET) is a molecular imaging modality that provides information at the molecular level. This system is composed of radiation detectors to detect incoming coincident annihilation gamma photons emitted from the radiopharmaceutical injected into a patient's body and uses these data to reconstruct images. A major trend in PET instrumentation is the development of time-of-flight positron emission tomography (ToF-PET). In ToF-PET, the time information (the instant the radiation is detected) is incorporated for image reconstruction. Therefore, precise and accurate timing recording is crucial in ToF-PET. ToF-PET leads to better localization of the annihilation event and thus results in overall improvement in the signal-to-noise ratio (SNR) of the reconstructed image. Several factors affect the timing performance of ToF-PET. In this article, the
\end{abstract}

Jung Yeol Yeom

jungyeol@korea.ac.kr

Muhammad Nasir Ullah

nasir@korea.ac.kr

Jimin Cheon

jimin.cheon@kumoh.ac.kr

Hojong Choi

hojongch@kumoh.ac.kr

1 Graduate School of Bio-Convergence Engineering, College of Health Sciences, Korea University, Seoul, South Korea

2 School of Electronic Engineering, Kumoh National Institute of Technology, Gumi, South Korea

3 Department of Medical IT Convergence Engineering, Kumoh National Institute of Technology, Gumi, South Korea

4 Department of Biomedical Engineering, Korea University, Seoul, South Korea background, early research and recent advances in ToF-PET instrumentation are presented. Emphasis is placed on the various types of scintillators, photodetectors and electronic circuitry for use in ToF-PET, and their impact on timing resolution is discussed.

Keywords Positron emission tomography · Time of flight . Instrumentation · Timing resolution

\section{Introduction}

Medical images are vital for the early detection and diagnosis of various diseases. Today, several types of imaging modalities, such as ultrasound, X-ray computed tomography (CT), magnetic resonance imaging (MRI) and scanners, are used in nuclear medicine. These imaging systems are used to acquire anatomical (structural), functional and molecular information regarding a subject [1]. Nuclear medicine employs technologies such as positron emission tomography (PET), single-photon-emitted computed tomography (SPECT) and gamma cameras to acquire predominantly molecular images, which are the most widely used molecular imaging tools in the clinic $[2,3]$.

During a PET scan, a short-life radioactive tracer isotope of known quantity incorporated into a biomolecule with an affinity to the area of interest is administrated into the living subject [4]. The most widely used radiopharmaceutical is the fluoro-deoxyglucose (FDG) as it gives information regarding glucose consumption at the cellular level [5]. The radioisotopes undergo positron emission decay in the process of becoming a stable element [6]. Each of these positrons travels a short distance within tissue before losing its kinetic energy and interacts with a neighboring electron [3]. As result of positron and electron annihilation reactions, a pair of gamma rays (photons) is produced in 
the opposite direction [7]. These anti-parallel rays are detected by detectors placed outside the patient's body, as shown in Fig. 1. The anti-parallel photons are recorded, and the virtual line connecting the two points is called the line of response (LoR) [2]. Based on these recorded data, the images are reconstructed by applying different image reconstruction algorithms [8].

PET images can be reconstructed with or without incorporated gamma photon time-of-flight (ToF) information [9]. In this article, the technique by which the image is reconstructed without using ToF information is called conventional PET and that incorporating ToF information is abbreviated as ToF-PET.

ToF information on emitted annihilation gamma photons before detection by the detector has been long known to potentially improve the overall performance of PET [3]. In the next section, the history and basic principal of ToF PET will be discussed, followed by a brief explanation of the time resolution of PET detectors. An overview of the ToF-PET detectors being studied for application in ToF-PET systems is also included in the article. Finally, the electronic considerations for ToF-PET are briefly described.

\section{Time-of-Flight Positron Emission Tomography (ToF-PET)}

\section{Physics of ToF-PET}

In a conventional PET system, positron annihilation is assumed to be localized somewhere along the LoR without information regarding the exact interaction point. Annihilation events along this LoR are thus considered to be evenly distributed along this line, adding noise to the image [10]. Contrarily, in ToF-PET, the ToF information (the detected time difference between the annihilation pairs) is used to localize the annihilation point to a smaller region along the LoR, improving the SNR of the final image. In Fig. 2, the difference between conventional PET and ToF-PET is illustrated. Figure 2 shows that the additional information provided by ToF-PET allows for much better localization of the original activity distribution of the radiopharmaceutical [11]. Figure 2a shows that in non-ToF reconstruction, information is weighted evenly along the whole LoR, while Fig. $2 b$ shows unwanted noise being subtracted with the help of ToF information as events can be restricted to a smaller region around the point of origin depending on the timing accuracy of the detector. ToF-PET uses the time difference $\Delta t$ in the detection of the photon pair, which travels in the opposite direction, and correlates it to position $\Delta x$ of the point of annihilation, as shown in Fig. 3a. If $c$ is the speed of light, the relation between times $\Delta t$ and $\Delta x$ can be shown as:

$\Delta x=\frac{c . \Delta t}{2}$

From the time difference $\Delta t$, one can estimate the annihilation position. Extremely fast detectors are needed to facilitate this very short time fraction [12]. Due to the limited time performance of the current detectors, it is not possible to localize the exact position of annihilation, which brings uncertainty to the registered data. As a result, the recorded time difference $\Delta t$ is blurred by a variance $\sigma_{\Delta x}^{2}$, resulting in a corresponding blurring in the estimated position $\Delta x$ by variance $\sigma_{\Delta x}^{2}$ [13], as shown in Fig. 3b. Improvement in the time performance of the ToF-PET system would enhance the gain in SNR of the registered data to provide better lesion detectability and/or reduce the scan time to improve patient throughput and/or decrease the patient dose to reduce patient exposure [14-16]. Equation (2) shows that the SNR of ToF-PET improves by a factor of approximately $\sqrt{\frac{D}{\Delta x}}$ compared to conventional PET.

$S N R_{T o F}=\sqrt{\frac{D}{\Delta x}} S N R_{n o n-T o F}$
Fig. 1 Illustration of the positron emission tomography principle

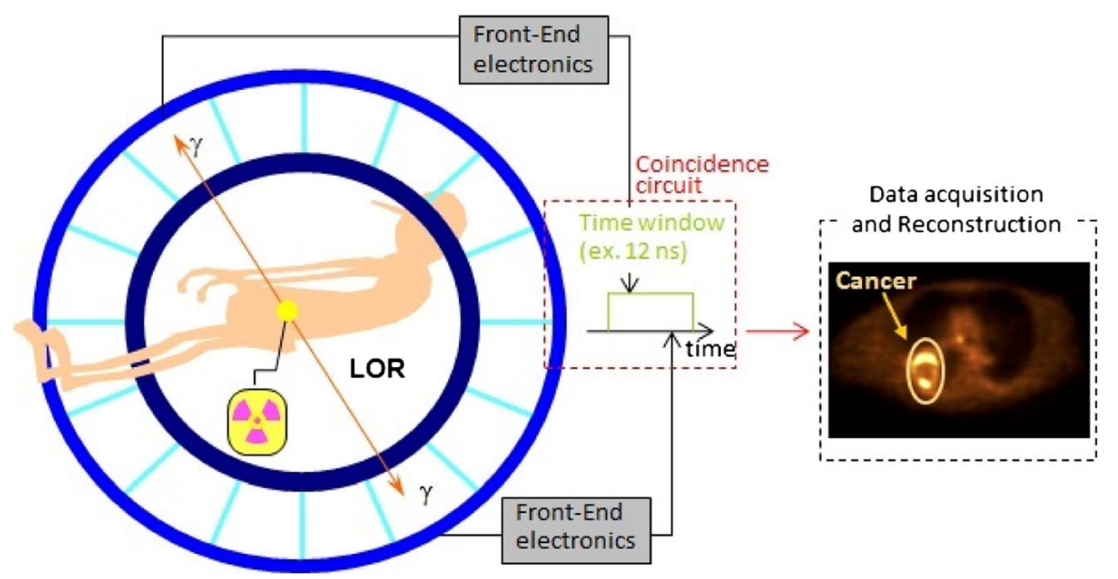


Fig. 2 Difference between conventional PET and ToF-PET [10]
Detector B

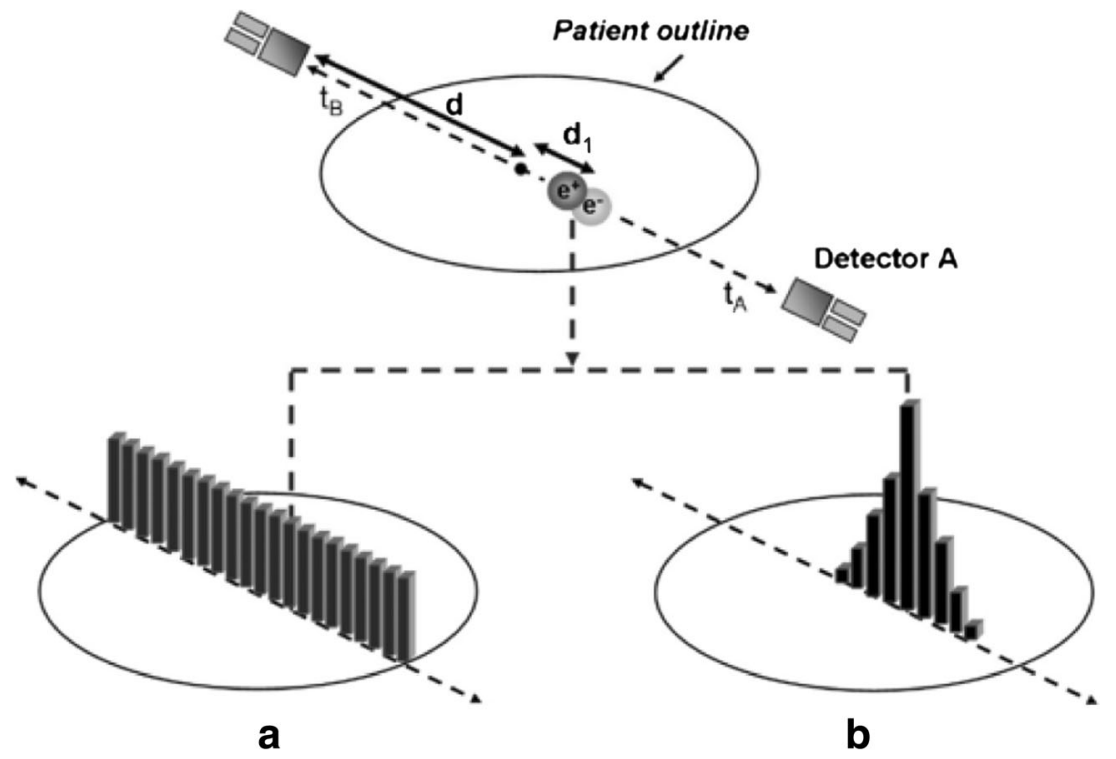

\section{Time Resolution}

The timing performance or accuracy of a PET detector is determined by its timing resolution. The timing (or temporal) resolution of the detector can be defined as its ability to record the minimum time difference between two subsequent photon events and differentiate between them efficiently [17]. In PET, the timing resolution is usually measured for a pair of detectors and reported as the full width half maximum (FWHM) of the distribution of the ToF difference between the detectors. The timing resolution of modern conventional PET systems ranges from $2 \mathrm{~ns}$ to $10 \mathrm{~ns}$, and commercial ToF-PET systems are known to achieve time resolution in the range of $500 \mathrm{ps}$ to 700 ps [13], but a timing resolution of 390 ps (per crystal timing performance) has been reported with a prototype commercial system [18]. Numerous studies with commercial systems have shown improved image quality [19], as shown in Fig. 4, and research is actively being carried out to further improve the timing performance of ToF-PET systems as described in the following sections.
Theoretically, the lowest limit in timing resolution can be estimated by a number of empirical formulas described in [21, 22]. The timing resolution of a ToF-PET system depends on a number of factors but is predominately determined by the detector and readout electronics. The details of individual detectors and their effect on timing resolution are discussed in the Choice of detectors for ToF-PET section.

\section{History and Early Research of ToF-PET}

ToF-PET is a technological advancement over conventional PET, and the idea was first implemented in the early 1980s [9, 23-25]. At that time, the three main groups involved in the development of ToF scanners were at CEA-LETI in France [26, 27], University of Washington St. Louis in Missouri, USA [28, 29], and the University of Texas, USA [30]. Other groups involved in ToF-PET research were the National Institute of Radiological Science in Chiba, Japan [31, 32], and Washington University in Seattle, WA, USA [33].
Fig. 3 Depiction of the ToF concept

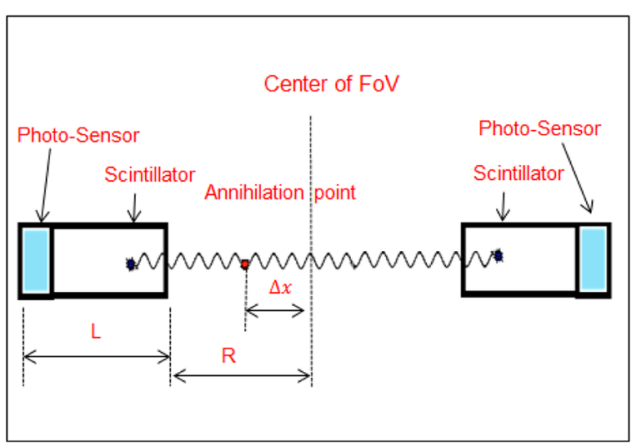

a

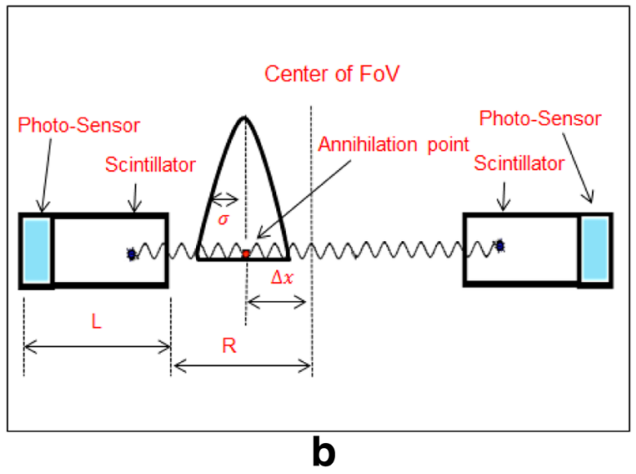




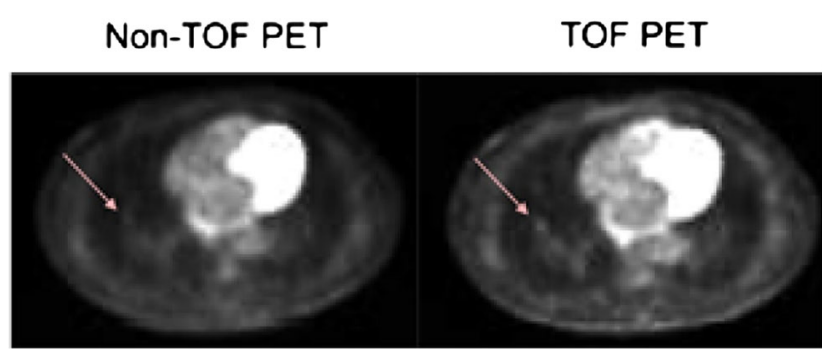

Lung lesion (3.5:1), BMI $=19$

Non-TOF PET TOF PET

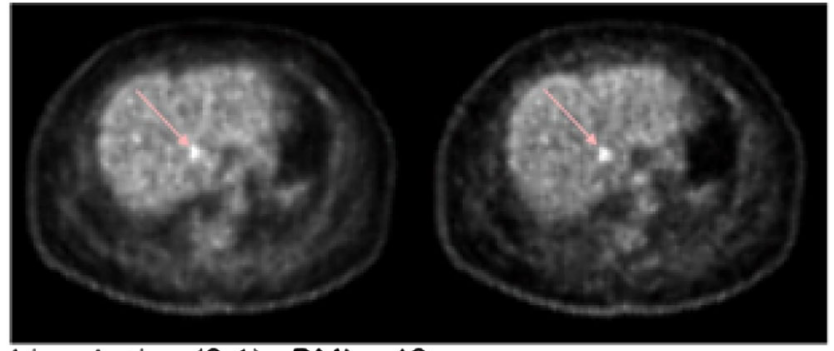

Liver lesion (2:1), BMI $=\mathbf{4 2}$

Fig. 4 Transverse images of lung lesions acquired with a system with a timing resolution of $670 \mathrm{ps}$ as reported in [20]

Cesium fluoride $(\mathrm{CsF})$ and barium fluoride $\left(\mathrm{BaF}_{2}\right)$ detectors were used in the first-generation TOF-PET [34-36]. Timing resolution of about $156 \mathrm{ps}$ and $212 \mathrm{ps}$ for $\mathrm{BaF}_{2}$ and $\mathrm{CsF}$, respectively, were achieved by Lewellen and colleagues. However, at the system level, time resolutions between $470 \mathrm{ps}$ and $750 \mathrm{ps}$ were achieved [34-38]. CsF needs careful packaging as it is highly hygroscopic. In addition, limited sensitivity (stopping power), low light output and the need for costly electronics restricted the first-generation ToF-PET to only research and consequently led to the demise of ToF-PET research at that time. During the 1970s and early 1980s, bismuth germanate $(\mathrm{BGO})$ was used as the standard scintillator for PET detectors because of its high detection efficiency and acceptable light output in commercial PET systems [39]. However, the detector based on this scintillator is not suitable for ToF-PET because of its low light output and relatively long decay time. Until recently, the unavailability of relevant technologies (scintillators, photosensors, electronics, etc.) stifled the progress of ToF-PET research.

\section{Choice of Detectors for ToF-PET}

PET detectors are predominately made up of two detector types: indirect or direct detectors of the annihilation gamma photons. In the latter, semiconductor detectors such as CZT and CdTe are used [40-44], but these detectors are reported to have poor timing resolution not suitable for ToF-PET [45]. Thus, in most ToF-PET detectors, the indirect detection method is adopted. Here, the incident radiation is detected through two steps of conversion. First, by using a scintillator, the annihilation radiation is converted into visible light, and in the next step, the visible light is converted into an electrical signal with the help of a photosensor (photodetector). Both steps contribute to the timing performance of PET detectors. The selections of these detectors are thus very crucial to the system resolution and performance. Other nonconventional detectors that have shown ToF's capabilities include gaseous detectors, such as resistive-plate chambers (RPC) [46], and concepts based on detection of prompt Cerenkov light are also being investigated [47].

\section{Scintillators for use in ToF-PET}

The scintillator characteristics that dominantly affect the detector's timing performance are the stopping power, light output (yield and wave length) and decay time. Ideally, a scintillator should be as dense as possible to detect all incoming radiation and give off high light output, and it should have a short decay time.

The discovery of newer scintillators such as lutetium orthosilicate $\left(\mathrm{Lu}_{2} \mathrm{SiO}_{2}\right)$ (LSO) [48], which showed promising properties such as high light output and a shorter decay time, crucial for ToF-PET, allowed researchers to enter into a new phase of ToF system development. These characteristics also made lutetium-based scintillators the standard detector in high-end commercial PET scanners [49, 50]. The shorter decay time of the scintillator also opened up possibilities for use in ToF-PET detectors. In the early phase development, Moses and colleagues achieved a time resolution of 300 ps by using a single LSO crystal in coincidence [51]. Initial reconstruction of ToF-PET using an LSO scanner in PET depicted a measurable gain in the image's SNR because of the use of ToF information in the reconstruction of the image even with a relatively poor time resolution of $1.2 \mathrm{~ns}[12,52]$. In recent years, LSO co-doped with $\mathrm{Ca}$ has also improved the timing performance compared to traditional LSO:Ce scintillators [10].

A commercial PET system name was introduced by Philips in 2006 [53] using LYSO:Ce crystals. The LYSO:Ce properties are very similar to those of LSO:Ce with only a slight difference in the densities. In LYSO:Ce crystals, a fraction of lutetium is replaced by yttrium, which has a lower density than lutetium. Most modern ToF-PET detectors, both commercial and research, are based mainly on LSO and LYSO crystals because of their comparatively high light yield, shorter decay time and manufacturability.

The discovery of cerium-doped lanthanum bromide $\left(\mathrm{LaBr}_{2}\right)$ [54] stirred excitement among researchers in the field because of its possibility to develop better ToF scanners. Despite the hygroscopic properties and lower stopping power compared to $\mathrm{L}(\mathrm{Y}) \mathrm{SO}, \mathrm{LaBr}_{2}$ has managed to produce promising results $[55,56]$. The first $\mathrm{LaBr}_{2}$ system is being developed at the University of Pennsylvania [57]. The 
time resolution measured for the system was $375 \mathrm{ps}$, but there is evidence that it can be brought down to $330 \mathrm{ps}[58,59]$.

In Table 1, comparisons between different scintillator types are shown. Other types of scintillators are available, such as $\mathrm{BaF}_{2}$ and plastic scintillators, but using them in TOF-PET may be challenging because of their low stopping power and low light output. The inorganic crystal $\mathrm{BaF}_{2}$ was first considered a very strong candidate for ToF-PET [62] because of its excellent time resolution, and different groups have reported achieving a timing resolution of less than 300 ps [35, 63]. However, the aforementioned shortcomings threw them out of the ToF-PET detector race. Recently, researchers have been working on a new type of crystal known as Ce:GAGG. Initial studies show that the timing performance is poorer than for $\mathrm{L}(\mathrm{Y}) \mathrm{SO}[60,64,65]$. However, a newer crystal in the family such as Ce:GFAG may be promising [66].

Other factors can also influence the timing performance. Besides the material constituents of the crystal, the shape and geometry of scintillators also contribute to the overall system resolution (see Sect. 2.4.6). The scintillator finishing also has to be taken into consideration [12]. A higher crystal reflective index decreases the light transmitted out of the crystal, which indirectly affects the system's time resolution. Finally, the wavelength of the emitted light highly affects the quantum efficiency of the subsequent photosensor.

\section{Photodetectors}

Even in the presence of ideal scintillators, one cannot achieve high system resolution without a matching photosensor. A good photosensor is one that has excellent quantum efficiency $(\mathrm{QE})$, a high gain and fast time response. The basic performance features of the different photosensors as reported in [67] and [68] are summarized in Table 2.

Photomultiplier Tubes (PMTs) The photomultiplier tube (PMT) is one of the various photosensor types and was considered the standard photodetector for ToF-PET because of its
Table 2 Comparative table of different photo-detectors used in PET [13]

\begin{tabular}{llll}
\hline & PMT & APD & SiPM \\
\hline Gain & $10^{6}$ & $50-1000$ & $10^{6}$ \\
Rise time (ns) & $\sim 1$ & $\sim 5$ & $\sim 1$ \\
QE @ $420 \mathrm{~nm}(\%)$ & $\sim 25$ & $\sim 70$ & $\sim 25-75(\mathrm{PDE})$ \\
Temperature sensitivity $\left(\% /{ }^{\circ} \mathrm{C}\right)$ & $<1$ & $\sim 3$ & $1-8$ \\
Bias (V) & $>1,000$ & $300-1,000$ & $30-80$ \\
Magnetic field sensitivity & yes & no & no \\
Sensitive area & $\mathrm{cm}^{2}$ & $\mathrm{~mm}^{2}$ & $\mathrm{~mm}^{2}$ \\
\hline
\end{tabular}

sub-nanosecond time resolution [3, 69]. PMTs have high gain and low noise, which reduces the uncertainty in the generated electrical signal with a small deviation from Poisson statistics [17]. Figure 5 shows the PMT and its constituents [3].

The PMT is a vacuum tube with a photocathode, several dynodes and an anode, which collectively produce extremely high gains to allow the detection of very low levels of light [70]. Scintillation photons enter into the PMT through the face plate where they deposit their energy at the cathode and eventually excite the electrons in the photocathode coating [3]. After the emission of electrons from the cathode, it is directed toward the dynodes with the help of an electric field [71]. Upon striking each dynode, the electrons multiply and are directed to the next dynode [72]. This process continues until the amplified electrons reach the anode.

The typical gain of PMTs is in the range of $\sim 10^{5}$ to $\sim 10^{7}$, which varies according to the applied voltage and number of dynode stages [73]. For ToF-PET, the most important requirements of PMTs are the QE and response time (including the transit time spread), which determine its timing resolution. The time resolution of PMTs can be defined as the FWHM of the distribution of the transit time spread $[74,75]$. The overall transit time of PMT is related to the variation of the electron transit time from the cathode to the first dynode. This variation occurs because of the different position of the electron emission from the photocathode and also depends on the

Table 1 Comparative table of fast scintillators that can be considered for ToF-PET [13, 60, 61]

\begin{tabular}{|c|c|c|c|c|c|c|}
\hline & $\mathrm{LSO}(\mathrm{Ce})$ & $\mathrm{LSO}(\mathrm{Ce}, \mathrm{Ca})$ & $\mathrm{LYSO}(\mathrm{Ce})$ & $\mathrm{LaBr}_{2}$ & Ce:GaGG & $\mathrm{LaBr}_{2}$ \\
\hline $\operatorname{Density}\left(\mathrm{g} / \mathrm{cm}^{3}\right)$ & 7.40 & Same as LSO & Same as LSO & 5.29 & 6.63 & 4.89 \\
\hline Effective atomic number(Z) & 66 & Same as LSO & Same as LSO & 46 & $>54$ & 54 \\
\hline Rise time (ns) & $\leq 0.5$ & Same as LSO & Same as LSO & $0.2-0.5$ & NA & $\sim 0.03$ \\
\hline Decay time (ns) & 40 & $31 @ 0.4 \% \mathrm{Ca}$ & Same as LSO & $15-26$ & 90 & $0.8 / 620$ \\
\hline Photon yield/KeV & $20-30$ & 35 & Same as LSO & 63 & 46 & $1.8 / 10$ \\
\hline Refractive index & 1.82 & 1.82 & 1.82 & 1.90 & NA & 1.56 \\
\hline Hygroscopic & No & No & No & Yes & No & Slightly \\
\hline Peak wave length (nm) & 420 & 420 & 420 & 380 & 520 & $220 / 310$ \\
\hline
\end{tabular}




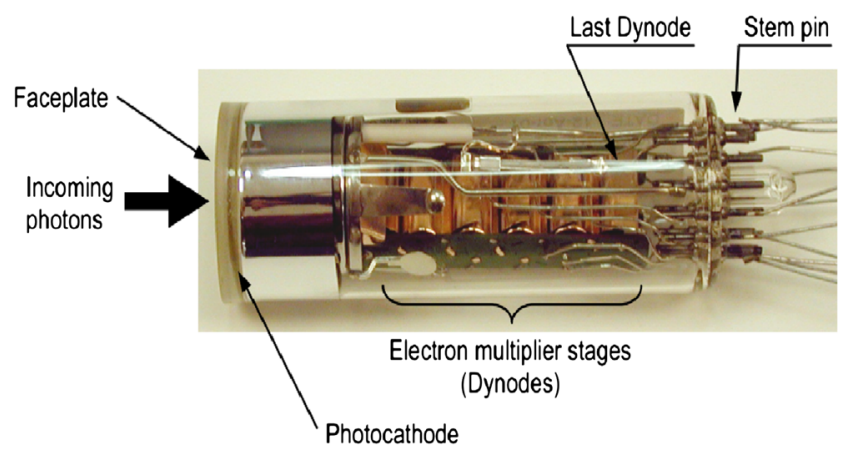

Fig. 5 Photomultiplier tube [13]

initial velocity of the emitted electrons. By increasing the potential difference between the cathode and first dynode, this variation can be reduced to some extent [3].

Overall, the time and energy resolution do not rely on a single entity but on the combination of different components, such as the scintillator detector, photodetector and electronics, and in fact on the mutual performance of all the components of that system. One of the best reported timing resolution on PMT-based PET detectors was by J.S. Karp [56]. The timing resolution achieved was 285 ps FWHM for single Ce-doped $\mathrm{LaBr}_{2}$ crystals and $290 \mathrm{ps}$ for an array of 100 crystals [56]. In 2011, Philips Medical Systems presented a ToF-PET/CT system using LYSO crystals with PMT that achieved a time resolution of $495 \mathrm{ps}$ at the system level. Some other groups also presented good results recently, such as [76] in 2006 and [77]. Moses et al. recorded and presented a time resolution of 218 ps FWHM for a single-ring detector module using an LYSO crystal in 2010 [78].

There are a few advantages of using PMTs in ToF-PET, as can be inferred from the table. PMTs are much less sensitive to temperature fluctuation compared to semiconductor detectors and have good gains but at the same time several drawbacks such as a low QE, relatively bulky size and need for a high voltage power supply [13].

There are several types of PMTs. Another type is the microchannel plate (MCP) PMT in which standard dynodes are replaced by a multichannel plate [79]. An MCP is composed of a number of small glass capillaries with diameters ranging from $\sim 2$ to $25 \mu \mathrm{m}$. Each glass capillary acts as an independent dynode, and a potential difference ( $\sim 800$ to $1400 \mathrm{~V})$ is applied across each plate [80]. A single multichannel plate gains around $\sim 10^{4}$, which can be increased by cascading them [3].

Compared to conventional PMT, an MCP-PMT typically has a faster response time and shorter transit time spread, and it is less susceptible to external magnetic fields and more compact. The performance results of MCP PMT are very encouraging, but it has some drawbacks such as low gain uniformity along the edges, low quantum QE and high cost [81].

Several groups have achieved promising results using MCP-PMTs. In 2010, Moses et al. used MCPs coupled with $24 \times 24$ pixelated LSO crystals and recorded coincidence timing resolution of 323 ps FWHM [81]. Kim et al. measured a timing resolution of 309 ps using a single $3 \mathrm{~mm} \times 3 \mathrm{~mm} \times 10 \mathrm{~mm}$ LYSO crystal-coupled MCP-PMT.

Avalanche Photodiode (APD) The avalanche photodiode (APD) is another type of photosensor that can be used in PET detectors. APDs have lower gain and response time compared to PMT, which make them unsuitable for ToF-PET [13].

Silicon Photomultiplier (SiPM) In recent years, the silicon photomultiplier (SiPM) appears to provide an alternative solution to APDs and PMTs as it combines the advantages of both. Table 2 shows that an SiPM has a comparable gain to a PMT with faster response time compared to an APD. SiPMs also operate in moderate bias voltage $(<100) \mathrm{V}$ [82], unlike PMTs, which operate at very high voltage. Finally, SiPMs are very compact in size and may be cheaper than PMTs when fabricated in bulk; they also have fewer aging issues [83].

A conventional SiPM is a made up of a many (several hundreds to thousands) microcells of $20 \mu \mathrm{m}$ to $100 \mu \mathrm{m}$ size affixed together on a common substrate, as shown in Fig. 6. SiPMs are also known as Geiger mode APDs (GAPDs), multipixel photon counters (MPPCs), etc., depending on the manufacturer or user. Within an SiPM pixel, each microcell responds independently to a photon interaction. When a
Fig. 6 Equivalent schematic of the structure and electronic schematic of the silicon photomultiplier $[84,85]$
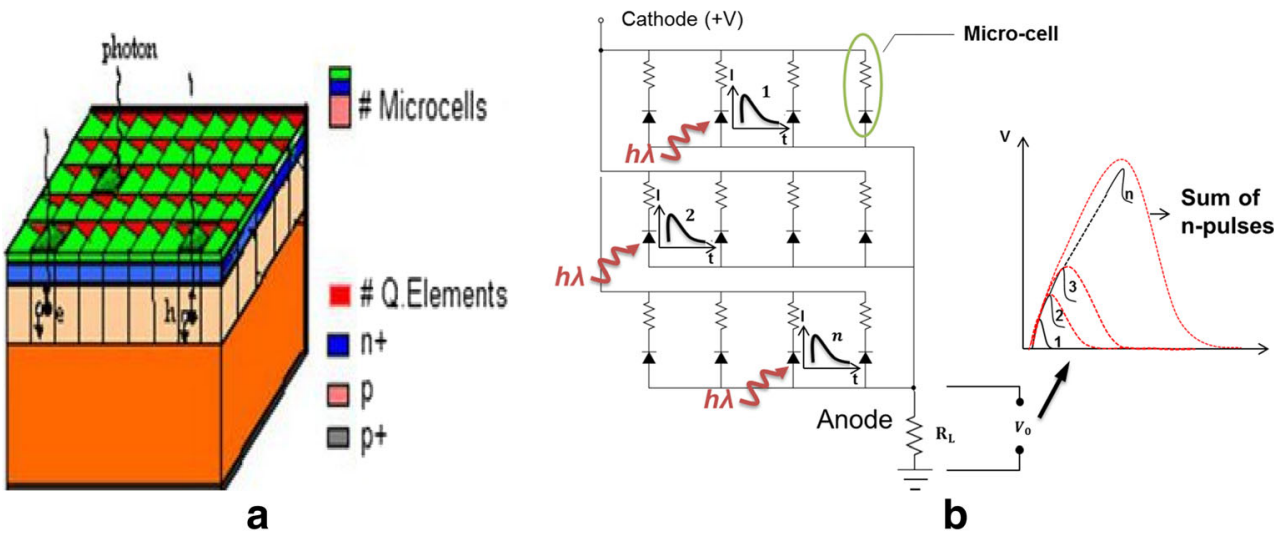
photon interacts with a microcell, it generates electron-hole pairs [86]. These electron-hole pairs trigger Geiger discharge when operated above the breakdown voltage [82]. The electrical equivalent circuit of SiPM can be seen in Fig. $6[84,85]$. Each microcell discharge is quenched with a small polysilicon load resistor that limits the current in each microcell to about $10 \mu \mathrm{A}$ [87]. The SiPM output signal is proportional to the number of breakdown cells fired as each individual cell operates like a binary device $[87,88]$.

The excellent intrinsic time resolution and higher photon detection efficiency (PDE) have made SiPM one of the most favorable devices for ToF-PET $[88,89]$ these days. In 2010, Schaart and colleagues published a research article in which they presented a coincidence timing resolution of $\sim 100 \mathrm{ps}$ FWHM using two $3 \mathrm{~mm} \times 3 \mathrm{~mm} \times 5 \mathrm{~mm} \mathrm{LaBr}_{2}$ :Ce (\%) crystals, each coupled with $3 \mathrm{~mm} \times 3 \mathrm{~mm}$ SiPM [55].

Fast readout electronics and signal processing are often required to extract information from conventional (analog) SiPM signals [90]. In order to overcome such shortcomings of analog SiPMs, a fully digital SiPM (dSiPM) was developed [91]. The difference between the analog SiPM and dSiPM is depicted in Fig. 7 [92]. While the timing performance is comparable to an analog SiPM, circuits such as counters and timeto-digital converters (see the Signal readout of Tof-PET detectors section) are integrated into the photosensor, negating the need to design electronics to read out SiPM signals, which in turn facilitates fast prototyping, but at the cost of higher power consumption and less room for customization. The dSiPM has fewer issues such as the electronic noise and temperature sensitivity drifts seen with analog SiPM [93]. Yeom et. al. achieved coincidence timing resolution (CRT) of $162 \pm 7 \mathrm{ps}$ FWHM with $3 \mathrm{~mm} \times 3 \mathrm{~mm} \times 20 \mathrm{~mm}$ LYSO crystals [94]. Other studies have shown promising results [95-97].

As stated above, the detector geometry (configuration) also affects its timing performance. Scintillators can be coupled to photosensors in several ways while still achieving ToF performance. Monolithic scintillators may provide both high spatial and timing resolution but require triggering at a very low photon level to achieve good timing resolution [98], while discrete coupling of individual scintillators is limited in spatial
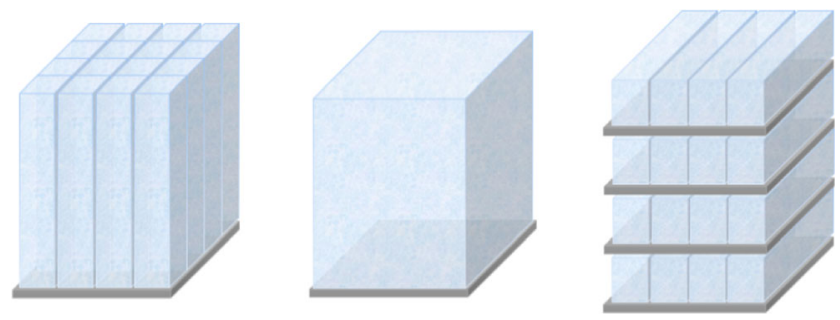

Fig. 8 Detector configurations for ToF-PET. Discrete crystals coupled to photosensors (left), a monolithic crystal on a photosensor array (center) and the stacked detector configuration (right)

resolution by their size $[90,95,99]$. The pro and cons of each configuration are discussed in [90]. Different types of detector configuration are shown in Fig. 8 .

\section{Signal Readout of Tof-PET Detectors}

The front-end readout circuit and electronic signal processing chain are vital parts of the PET system. Required characteristics for PET electronics are: (1) a large number of readout channels, (2) high bandwidth and low noise and (3) a high count rate [100]. In addition to these requirements, the cost and power consumption should be considered [101]. The front-end electronics typically comprise analog circuits to process the detector signals, which are then passed on to the backend data acquisition (DAQ) board for further processing. Due to the large number of channels required in PET, the front-end electronics are often designed using application-specific integrated circuit (ASIC) technologies (nino, petrioc), although they can also be fabricated with discrete components for smaller systems [101, 102].

Typical electronic signal processing begins at the photosensor. The electrical signal generated by a photosensor is often read out with a preamplifier. Preamplifiers serve three main purposes: (1) to amplify the signal if required, (2) to match the impedance level between the detector and subsequent components and (3) to shape the signal for optimal processing [103]. Most preamplifiers for radiation detectors are fabricated in the form of charge- or current-sensitive configuration [90, 104-106]. In terms of noise, the former design is superior, but in ToF-PET where high-gain
Fig. 7 Scintillation light detector systems based on the analog (a) and digital (b) silicon photomultiplier [92]

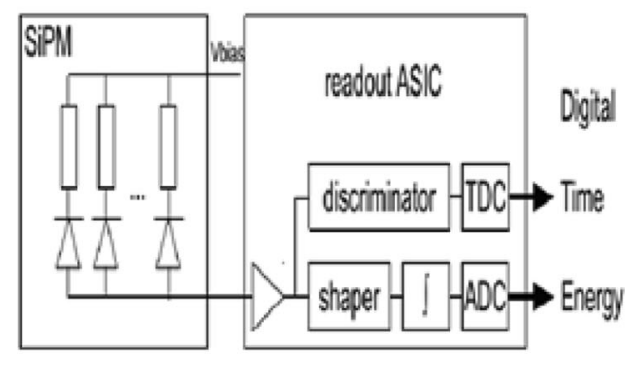

a

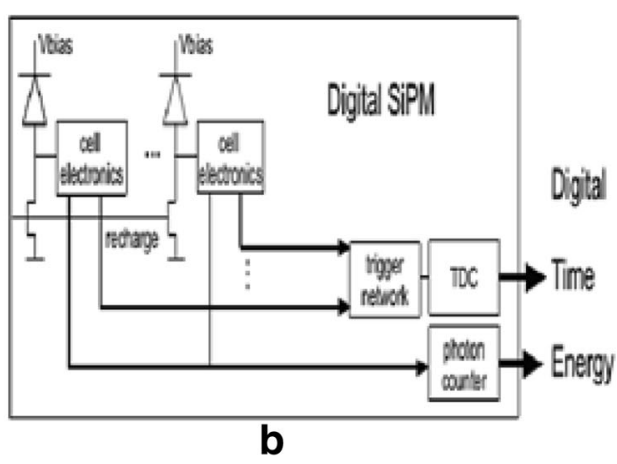




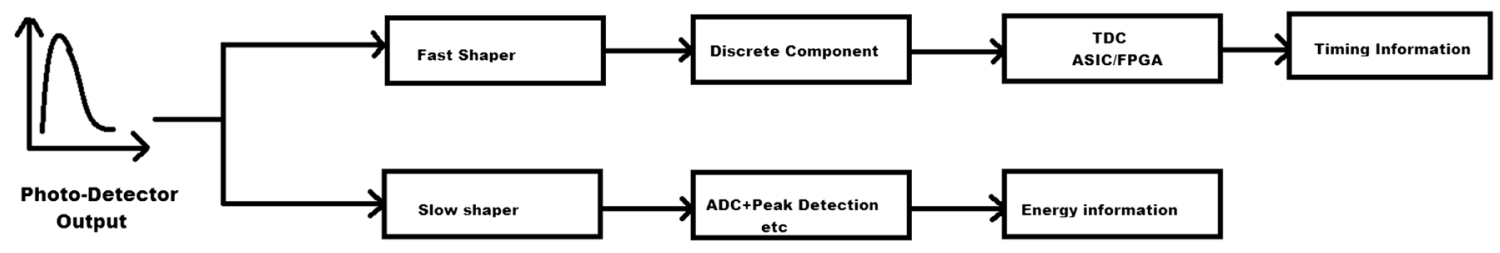

Fig. 9 Conventional method to extract timing and energy information from the photosensor output signal

photosensors are used, a relatively large feedback capacitor is required, which decreases the response time and is difficult to implement with ASIC technology. Most preamplifiers for ToF applications therefore adopt the latter configuration [107, 108].

Signal processing begins after the preamplifier circuit. The information to be extracted from the signal is the energy, position and time of detection (timestamp). The most straightforward method would be to digitize the preamplifier signal at an early stage and acquire all required information from the waveform at the PC [109]. This method, however, is costly to implement, especially for ToF-PET where high-speed $\mathrm{GHz}$ digitizers are required to sufficiently sample the fast signals $[104,110]$.

Therefore, most PET systems employ an approach similar to that shown in Fig. 9 where the signal is split in different paths for extracting energy and time information, i.e., a fast path where a fast shaping filter circuit is used to preserve the fast component of the detector signal for timing information and a slow path where a band-pass filter is used to remove unwanted noise and shape the pulse for digitization with a slow ADC to acquire energy information. In ToF measurement, acquiring the exact arrival times of photons is very important. Different time pick-off methods, such as leading edge timing, cross-over timing or constant fraction discrimination (CFD), can be used to record the photon arrival time. The leading edge method is very direct; once the signal has crossed the fixed threshold level, a trigger signal is generated but can give rise to timing errors such as time walk or time jitter [3]. The cross-over timing method uses zero crossing of signal for time measurements, which compensates for errors arising from amplitude walk but requires bipolar signals [102]. In ToF-PET, however, leading edge triggering at very low photon counts has been reported to provide excellent timing performance in numerous studies [111, 112]. A comparator circuit triggered on the rising edge is often used for this purpose.

Timing and energy information can be acquired by using either analog circuitry [102] or digital circuits such as the ASICs or field programmable gate array (FPGA). Many drawbacks are associated with using analog circuits, such as the relatively complex design, high power dissipation and bulkiness. On the other hand, analog ASIC designs are more complex and have long design cycles. Therefore, numerous studies are being carried out using FPGA for the time-to-digital converter (TDCs) circuit $[113,114]$. These
TDCs provide the solution to the shortcoming of the analog approach [100, 102]. Most TDCs are designed on ASICs, which are very expensive and on the other hand have a limited number of channels and functionality. Reconfiguration capabilities and low cost make FPGAs highly suited for the ToF electronic signal processing chain, and they also have the ability to measure the ToF with good resolution. Recent advances in digital electronic design paved the way for FPGA to be used for TOF measurement with promising jitter of less than $20 \mathrm{ps}$ [114-121].

At the end of the signal processing chain, a data acquisition (DAQ) system collects and processes the aforementioned data for image reconstruction in the host computer [122]. An open source electronics system that can be used for data acquisition of nuclear medicine modalities is the OpenPET project [100].

\section{Conclusions}

ToF-PET has the potential to greatly improve the performance of conventional PET systems. The timing resolutions of PET detectors have significantly improved since research began in the 1980s. Nonetheless, there is still room for improvement in ToF-PET systems. The discoveries of new scintillation crystals, better photosensors and advancement in electronics technology have reignited interest in ToF systems, and the timing performance can be expected to continue gradually improving in the near future.

Acknowledgments This work is supported in part by the National Research Foundation of Korea (NRF-2014R1A1A1005242), MSIP (Ministry of Science, ICT and Future Planning), Korea, under the C-ITRC (Convergence Information Technology Research Center) (IITP-2015-H8601-15-1011) supervised by the IITP (Institute for Information \& Communications Technology Promotion) and Quality of Working Life (QWL) (N0000842) Program funded by the Ministry of Trade, Industry \& Energy of Korea.

\section{Compliance with Ethical Standards}

Conflict of Interest M.Nasir Ullah, Eva Pratiwi, Jimin Cheon, Hojong Choi and Jung Yeol Yeom declare that they have no conflict of interest.

Ethical Statement This article does not contain any studies with human participants or animals performed by any of the authors. This study has been performed in accordance with the ethical standards laid down in the 1964 Declaration of Helsinki and its later amendments. 


\section{References}

1. Ollinger JM. Fessler JA Positron-Emission Tomography. IEEE Signal Process Mag. 1997;14(1):43-55.

2. Rouze N, Winkle W, Hutchin G. IndyPET - a high resolution, high sensitivity dedicated research scanner. Nuclear Science Symposium, 1999 Conference Record 1999 IEEE: IEEE; 1999. pp. 1460-4.

3. Bauer F. Detector considerations for time-of-flight in positron emission tomography. 2009.

4. Muehllehner G, Karp JS. Positron emission tomography. Phys Med Biol. 2006;51:R117.

5. Bailey DL, Townsend DW, Valk PE, Maisey MN. Positron emission tomography. Springer; 2005.

6. Zanzonico P. Positron emission tomography: a review of basic principles, scanner design and performance, and current systems. In Seminars in nuclear medicine. 2004;34(2):87-111.

7. Valk PE, Delbeke D, Bailey DL, Townsend DW, Maisey MN. Positron emission tomography clinical pratice. 2003. SpringerVerlag London Limited. doi:10.1007/1-84628-187-3.

8. Leahy RM, Qi J. Statistical approaches in quantitative positron emission tomography. Stat Comput. 2000;10:147-65.

9. Budinger TF. Time-of-flight positron emission tomography: status relative to conventional PET. J Nucl Med. 1983;24:73-8.

10. Moses WW. Recent advances and future advances in time-offlight PET. Nucl Instrum Methods PhysRes, Sect A. 2007;580: 919-24.

11. Townsend D. Multimodality imaging of structure and function. Phys Med Biol. 2008;53:R1.

12. Conti M. State of the art and challenges of time-of-flight PET. Phys Med. 2009;25:1-11.

13. Spanoudaki VC, Levin CS. Photo-detectors for time of flight positron emission tomography (ToF-PET). Sensors. 2010;10: 10484-505.

14. Murray I, Kalemis A, Glennon J, Hasan S, Quraishi S, Beyer T, et al. Time-of-flight PET/CT using low-activity protocols: potential implications for cancer therapy monitoring. Eur J Nucl Med Mol Imaging. 2010;37:1643-53.

15. Karp JS, Surti S, Daube-Witherspoon ME, Muehllehner G. Benefit of time-of-flight in PET: experimental and clinical results. J Nucl Med. 2008;49:462-70.

16. Surti S, Karp JS, Popescu LM, Daube-Witherspoon ME, Werner $M$. Investigation of time-of-flight benefit for fully 3-DPET. IEEE Trans Med Imaging. 2006;25:529-38.

17. Knoll GF. Radiation detection and measurement. 4th ed. USA: John Wiley \& Sons; 2010.

18. Levin C, Glover G, Deller T, McDaniel D, Peterson W, Maramraju SH. Prototype time-of-flight PET ring integrated with a 3T MRI system for simultaneous whole-body PET/MR imaging. J Nucl Med. 2013;54:148.

19. Surti S, Scheuermann J, El Fakhri G, Daube-Witherspoon ME, Lim R, Abi-Hatem N, et al. Impact of time-of-flight PET on whole-body oncologic studies: a human observer lesion detection and localization study. J Nucl Med. 2011;52:712-9.

20. El Fakhri G, Surti S, Trott CM, Scheuermann J, Karp JS. Improvement in lesion detection with whole-body oncologic timeof-flight PET. J Nucl Med. 2011;52:347-53.

21. Paulus T. Principles and applications of timing spectroscopy. Application Note AN-42, EG\&E; 1982.

22. Bell R. Comparison of leading-edge and crossover timing in coincidence measurements. Nucl Inst Methods. 1966;42:211-2.

23. Budinger TF. Instrumentation trends in nuclear medicine. Semin Nucl Med. 1977;7(4):285-97.

24. Mullani NA, Markham J, Ter-Pogossian MM. Feasibility of timeof-flight reconstruction in positron emission tomography. J Nucl Med. 1980;21:1095-7.
25. Gariod R, Allemand R, Cormoreche E, Laval M, Moszynski M. The LETI positron tomograph architecture and time of flight improvements. Proceedings of The Workshop on Time of Flight Tomography 1982. p. 25-9.

26. Bendriem B, Soussaline F, Campagnolo R, Verrey B, Wajnberg P, Syrota A. A technique for the correction of scattered radiation in a PET system using time-of-flight information. J Comput Assist Tomogr. 1986;10:287-95.

27. Ter-Pogossian MM, Ficke DC, Yamamoto M, Hood Sr JT. Super PETT I: a positron emission tomograph utilizing photon time-offlight information. IEEE Trans Med Imaging. 1982;1:179-87.

28. Yamamoto M, Ficke DC, Ter-Pogossian MM. Experimental assessment of the gain achieved by the utilization of time-of-flight information in a positron emission tomograph (super PETT I). IEEE Trans Med Imaging. 1982;1:187-92.

29. Wong W-H, Mullani NA, Philippe EA, Hartz R, Gould KL. Image improvement and design optimization of the time-of-flight PET. J Nucl Med. 1983;24:52-60.

30. Wong W, Mullani N, Philippe E, Hartz R, Bristow D, Yerian K, et al. Performance characteristics of the University of Texas TOFPET-I PET camera. J Nucl Med. 1986;25.

31. Tomitani T. Image reconstruction and noise evaluation in photon time-of-flight assisted positron emission tomography. IEEE Trans Nucl Sci. 1981;28:4581-9.

32. Yamamoto M, Nohara N, Tanaka E, Tomitani T, Murayama H, Sato N, et al. Time-of-flight positron imaging and the resolution improvement by an iterative method. IEEE Trans Nucl Sci. 1989;36:998-1002.

33. Lewellen T, Bice A, Harrison R, Pencke M, Link J. Performance measurements of the SP3000/UW time-of-flight positron emission tomograph. IEEE Trans Nucl Sci. 1988;35.

34. Allemand R, Gresset C, Vacher J. Potential advantages of a cesium fluoride scintillator for a time-of-flight positron camera. J Nucl Med. 1980;21:153-5.

35. Laval M, Moszyński M, Allemand R, Cormoreche E, Guinet P, Odru R, et al. Barium fluoride-inorganic scintillator for subnanosecond timing. Nucl Instrum Methods PhysRes, Sect A. 1983;206:169-76.

36. Ishii K, Orihara H, Matsuzawa T, Binkley DM, Nutt R. High resolution time-of-flight positron emission tomograph. Rev Sci Instrum. 1990;61:3755-62.

37. Soussaline F, Comar D, Allemand R, Campagnolo R, Laval M, Vacher J. New developments in positron emission tomography instrumentation using the time-of-flight information, The metabolism of the human brain studied with positron emission tomography. New York: Raven Press; 1985. p. 1-11.

38. Lewellen TK. Time-of-flight PET. Semin Nucl Med. 1988;28(3): 268-75.

39. Weber MJ, Monchamp RR. Luminescence of Bi4 Ge3 O12: spectral and decay properties. J Appl Phys. 1973;44:5495-9.

40. Cates JW, Gu Y, Levin CS. Direct conversion semiconductor detectors in positron emission tomography. Mod Phys Lett A. 2015;30:1530011.

41. Matteson JL, Gu Y, Skelton RT, Deal AC, Stephan EA, Duttweiler F, Huszar GL, Gasaway TM, Levin CS. Charge collection studies of a high resolution CZT-based detector for PET. Nuclear Science Symposium Conference Record, 2008. NSS '08. IEEE. 19-25 Oct 2008. pp 503-510. Dresden, Germany. doi:10.1109/ NSSMIC.2008.4775215.

42. Scheiber C, Giakos GC. Medical applications of CdTe and CdZnTe detectors. Nucl Instrum Methods PhysRes, Sect A. 2001;458:12-25.

43. Eisen Y, Shor A, Mardor I. CdTe and CdZnTe gamma ray detectors for medical and industrial imaging systems. Nucl Instrum Methods PhysRes, Sect A. 1999;428:158-70. 
44. Del Sordo S, Abbene L, Caroli E, Mancini AM, Zappettini A, Ubertini P. Progress in the development of CdTe and CdZnTe semiconductor radiation detectors for astrophysical and medical applications. Sensors. 2009;9:3491-526.

45. Gu Y, Matteson J, Skelton R, Deal A, Stephan E, Duttweiler F, et al. Study of a high-resolution, 3D positioning cadmium zinc telluride detector for PET. Phys Med Biol. 2011;56:1563.

46. Georgiev G, Ilieva N, Kozhuharov V, Lessigiarska I, Litov L, Pavlov B, et al. Multigap RPC for PET: development and optimisation of the detector design. J Instrum. 2013;8, P01011.

47. Korpar S, Dolenec R, Križan P, Pestotnik R, Stanovnik A. Study of TOF PET using Cherenkov light. Phys Procedia. 2012;37: 1531-6.

48. Melcher C, Schweitzer J. Cerium-doped lutetium oxyorthosilicate: a fast, efficient new scintillator. IEEE Trans Nucl Sci. 1992;39: $502-5$.

49. Wienhard K, Schmand M, Casey M, Baker K, Bao J, Eriksson L, et al. The ECAT HRRT: performance and first clinical application of the new high resolution research tomograph. IEEE Trans Nucl Sci. 2002:49:104-10.

50. Herzog H, Tellmann L, Hocke C, Pietrzyk U, Casey ME, Kuwert T. NEMA NU2-2001 guided performance evaluation of four Siemens ECAT PET scanners. IEEE Trans Nucl Sci. 2004;51: 2662-9.

51. Moses WW, Derenzo S. Prospects for time-of-flight PET using LSO scintillator. IEEE Trans Nucl Sci. 1999;46:474-8.

52. Conti M, Bendriem B, Casey M, Chen M, Kehren F, Michel C, et al. Implementation of time-of-flight on CPS HiRez PET scanner. Nuclear Science Symposium Conference Record, 2004 IEEE: IEEE; 2004. p. 2796-800.

53. Surti S, Kuhn A, Werner ME, Perkins AE, Kolthammer J, Karp JS. Performance of Philips Gemini TF PET/CT scanner with special consideration for its time-of-flight imaging capabilities. J Nucl Med. 2007;48:471-80.

54. Conti M, Bendriem B, Casey M, Chen M, Kehren F, Michel C, et al. First experimental results of time-of-flight reconstruction on an LSO PET scanner. Phys Med Biol. 2005;50:4507.

55. Schaart DR, Seifert S, Vinke R, van Dam HT, Dendooven P, Löhner H, et al. LaBr3: Ce and SiPMs for time-of-flight PET: achieving 100 ps coincidence resolving time. Phys Med Biol. 2010;55:N179.

56. Kuhn A, Surti S, Karp J, Raby P, Shah K, Perkins A, et al. Design of a lanthanum bromide detector for time-of-flight PET. IEEE Trans Nucl Sci. 2004;51:2550-7.

57. Karp J, Kuhn A, Perkins A, Surti S, Werner M, Daube-Witherspoon $\mathrm{M}$, et al. Characterization of a time-of-flight PET scanner based on lanthanum bromide. Nuclear Science Symposium Conference Record, 2005 IEEE: IEEE; 2005. p. 5 pp.

58. Kyba C, Wiener R, Newcomer F, Van Berg R, Dressnandt N, Karp JS. Timing measurements from a TOF-PET scanner using local PMT triggering. Nuclear Science Symposium Conference Record, 2007 NSS'07 IEEE: IEEE; 2007. p. 4123-8.

59. Daube-Witherspoon M, Surti S, Perkins A, Kyba C, Wiener R, Werner M, et al. The imaging performance of a LaBr3-based PET scanner. Phys Med Biol. 2010;55:45.

60. Yeom JY, Yamamoto S, Derenzo SE, Spanoudaki VC, Kamada K, Endo T, et al. First performance results of Ce: GAGG scintillation crystals with silicon photomultipliers. IEEE Trans Nucl Sci. 2013;60:988-92.

61. Kurosawa S, Kamada K, Yokota Y, Yoshikawa A. Luminescent properties of Ce: $\mathrm{Gd} 3$ (Al, Ga, Mg, M) $5 \mathrm{O} 12$ crystal (M= Zr, Hf). Jpn J Appl Phys. 2014;53, 04EG14.

62. Derenzo S, Weber M, Moses W, Dujardin C. Measurements of the intrinsic rise times of common inorganic scintillators. Nuclear Science Symposium, 1999 Conference Record 1999 IEEE: IEEE; 1999. p. 152-6.
63. Ziegler S, Ostertag H, Kuebler WK, Lorenz WJ, Otten EW. Effects of scintillation light collection on the time resolution of a time-of-flight detector for annihilation quanta. IEEE Trans Nucl Sci. 1990;37:574-9.

64. Ferri A, Gola A, Serra N, Tarolli A, Zorzi N, Piemonte C. Performance of FBK high-density SiPM technology coupled to Ce: LYSO and Ce: GAGG for TOF-PET. Phys Med Biol. 2014;59:869.

65. Yamamoto S, Yeom JY, Kamada K, Endo T, Levin CS. Development of an ultrahigh resolution block detector based on $0.4 \mathrm{~mm}$ pixel Ce: GAGG scintillators and a silicon photomultiplier array. IEEE Trans Nucl Sci. 2013;60:4582-7.

66. Kamada K, Nikl M, Kurosawa S, Beitlerova A, Nagura A, Shoji $\mathrm{Y}$, et al. Alkali earth co-doping effects on luminescence and scintillation properties of Ce doped $\mathrm{Gd} 3 \mathrm{Al} 2 \mathrm{Ga} 3 \mathrm{O} 12$ scintillator. Opt Mater. 2015;41:63-6.

67. Renker D. Geiger-mode avalanche photodiodes, history, properties and problems. Nucl Instrum Methods PhysRes, Sect A 2006;567:48-56.

68. Wernick MN, Aarsvold JN. Emission tomography: the fundamentals of PET and SPECT. Academic Press; 2004.

69. Moszyński M, Kapusta M, Nassalski A, Szcześniak T, Wolski D, Eriksson L, et al. New prospects for time-of-flight PET with LSO scintillators. Nuclear Science Symposium Conference Record, 2005 IEEE: IEEE; 2005. p. 2854-8.

70. Haselman M, Miyaoka R, Lewellen TK, Hauck S. Fpga-based data acquisition system for a positron emission tomography (PET) scanner. FPGA2008. p. 264.

71. Flower MA. Webb's physics of medical imaging. CRC Press; 2012.

72. Green D. The physics of particle detectors. Cambridge: University Press; 2000.

73. Bauer F, Zhang N, Schmand M, Loope M, Eriksson L, Aykac M. Dynode-timing method for PET block detectors. IEEE Trans Nucl Sci. 2008;55:451-6.

74. Hamamatsu Photonics K. Photomultiplier tubes-basics and applications, 3rd eddition. Hamamatsu Photonics; 2006.

75. Flyckt S-O. Photomultiplier tubes: principles and applications. Photonis; 2002.

76. Kuhn A, Surti S, Karp J, Muehllehner G, Newcomer F, VanBerg R. Performance assessment of pixelated $\mathrm{LaBr} 3$ detector modules for time-of-flight PET. IEEE Trans Nucl Sci. 2006;53:1090-5.

77. Szczęśniak T, Moszyński M, Nassalski A, Lavoute P, Kapusta M. Fast photomultipliers for TOF PET. Nuclear Science Symposium Conference Record, 2007 NSS'07 IEEE: IEEE; 2007. p. 2651-9.

78. Moses W, Janecek M, Spurrier M, Szupryczynski P, Choong W-S, Melcher C, et al. Optimization of a LSO-based detector module for time-of-flight PET. IEEE Trans Nucl Sci. 2010;57:1570-6.

79. Kume H, Koyama K, Nakatsugawa K, Suzuki S, Fatlowitz D. Ultrafast microchannel plate photomultipliers. Appl Opt. 1988;27: $1170-8$.

80. Anashin V, Beschastnov P, Golubev V, Mironenko L, Salnikov A, Serednyakov S. Photomultipliers with microchannel plates. Nucl Instrum Methods PhysRes, Sect A. 1995;357:103-9.

81. Kim H, Frisch H, Chen C-T, Genat J-F, Tang F, Moses W, et al. A design of a PET detector using micro-channel plate photomultipliers with transmission-line readout. Nucl Instrum Methods PhysRes, Sect A. 2010;622:628-36.

82. Buzhan P, Dolgoshein B, Filatov L, Ilyin A, Kantzerov V, Kaplin $\mathrm{V}$, et al. Silicon photomultiplier and its possible applications. Nucl Instrum Methods PhysRes, Sect A. 2003;504:48-52.

83. Henseler D, Grazioso R, Zhang N, Schmand M. SiPM performance in PET applications: An experimental and theoretical analysis. Nuclear Science Symposium Conference Record (NSS/MIC), 2009 IEEE: IEEE; 2009. p. 1941-8. 
84. Saveliev V. Silicon photomultiplier-new era of photon detection. INTECH Open Access Publisher; 2010.

85. Yeom JY, Vinke R, Pavlov N, Bellis S, Wall L, O'Neill K, et al. Fast timing silicon photomultipliers for scintillation detectors. IEEE Photon Technol Lett. 2013;25:1309-12.

86. Piemonte C. A new silicon photomultiplier structure for blue light detection. Nucl Instrum Methods PhysRes, Sect A. 2006;568:224-32.

87. Dolgoshein B, Balagura V, Buzhan P, Danilov M, Filatov L, Garutti E, et al. Status report on silicon photomultiplier development and its applications. Nucl Instrum Methods PhysRes, Sect A. 2006;563:368 76.

88. Roncali E, Cherry SR. Application of silicon photomultipliers to positron emission tomography. Ann Biomed Eng. 2011;39:1358-77.

89. Del Guerra A, Belcari N, Bisogni MG, LLosa G, Marcatili S, Ambrosi G, et al. Advantages and pitfalls of the silicon photomultiplier (SiPM) as photodetector for the next generation of PET scanners. Nucl Instrum Methods PhysRes, Sect A. 2010;617:223-6.

90. Yeom JY, Vinke R, Levin CS. Optimizing timing performance of silicon photomultiplier-based scintillation detectors. Phys Med Biol. 2013;58:1207.

91. Haemisch Y, Frach T, Degenhardt C, Thon A. Fully digital arrays of silicon photomultipliers (dSiPM)-a scalable alternative to vacuum photomultiplier tubes (PMT). Phys Procedia. 2012;37:1546-60.

92. Frach T, Prescher G, Degenhardt C, De Gruyter R, Schmitz A, Ballizany R. The digital silicon photomultiplier-Principle of operation and intrinsic detector performance. Nuclear Science Symposium Conference Record (NSS/MIC), 2009 IEEE: IEEE; 2009. p. 1959-65.

93. Degenhardt C, Prescher G, Frach T, Thon A, De Gruyter R, Schmitz A, et al. The digital silicon photomultiplier - a novel sensor for the detection of scintillation light. Nuclear Science Symposium Conference Record (NSS/MIC), 2009 IEEE: IEEE; 2009. p. 2383-6.

94. Yeom JY, Vinke R, Bieniosek MF, Levin CS. Comparison of end/ side scintillator readout with digital-SiPM for ToF PET. Nuclear Science Symposium and Medical Imaging Conference (NSS/ MIC), 2013 IEEE: IEEE; 2013. p. 1-3.

95. Joram C, Collaboration A-P. Imaging results and TOF studies with axial PET detectors. Nucl Instrum Methods PhysRes, Sect A. 2013;732:586-90.

96. Marcinkowski R, Espana S, Thoen H, Vandenberghe S. Performance of digital silicon photomultipliers for time of flight PET scanners. Nuclear Science Symposium and Medical Imaging Conference (NSS/MIC), 2012 IEEE: IEEE; 2012. p. 2825-9.

97. Marcinkowski R, España S, Van Holen R, Vandenberghe S. Optimized light sharing for high-resolution TOF PET detector based on digital silicon photomultipliers. Phys Med Biol. 2014;59:7125-39.

98. Schaart DR, van Dam HT, Seifert S, Vinke R, Dendooven P, Löhner H, et al. A novel, SiPM-array-based, monolithic scintillator detector for PET. Phys Med Biol. 2009;54:3501.

99. Casella C, Heller M, Joram C, Schneider T. A high resolution tofpet concept with axial geometry and digital sipm readout. Nucl Instrum Methods PhysRes, Sect A. 2014;736:161-8.

100. Moses W, Buckley S, Vu C, Peng Q, Pavlov N, Choong W-S, et al. OpenPET: a flexible electronics system for radiotracer imaging. IEEE Trans Nucl Sci. 2010;57:2532-7.

101. Hong KJ, Choi Y, Jung JH, Kang J, Hu W, Lim HK, et al. A prototype MR insertable brain PET using tileable GAPD arrays. Med Phys. 2013;40:042503.

102. Torres J, García R, Aguilar A, Soret J, Martos J, González A, et al. Implementation of TOF-PET Systems on Advanced Reconfigurable Logic Devices. doi:10.5772/57123.

103. Cherry SR, Sorenson JA, Phelps ME. Physics in nuclear medicine. Elsevier Health Sciences; 2012.
104. Yeom JY, Vinke R, Spanoudaki VC, Hong KJ, Levin CS. Readout electronics and data acquisition of a positron emission tomography time-of-flight detector module with waveform digitizer. IEEE Trans Nucl Sci. 2013;60:3735-41.

105. McElroy DP, Pimpl W, Pichler BJ, Rafecas M, Schüler T, Ziegler S. Characterization and readout of MADPET-II detector modules: validation of a unique design concept for high resolution small animal PET. IEEE Trans Nucl Sci. 2005;52:199-204.

106. Rolo M, Alves L, Martins E, Rivetti A, Santos M, Varela J. A lownoise CMOS front-end for TOF-PET. J Instrum. 2011;6, P09003.

107. Gao W, Gao D, Wei T, Hu Y. Advances in front-end readout ASIC design for PET imaging. Solid-State and Integrated Circuit Technology (ICSICT), 2012 I.E. 11th International Conference on: IEEE; 2012. p. 1-4.

108. Yeom J, Ishitsu T, Takahashi H. Development of a waveform sampling front-end ASIC for PET. Proceedings of the 2004 Asia and South Pacific Design Automation Conference. IEEE Press; 2004. p. 567-8.

109. Yeom J, Shimazoe K, Takahashi H, Murayama H. A waveform sampling front-end ASIC for readout of GSO/APD with DOI information. Nucl Instrum Methods PhysRes, Sect A. 2007;571:381-4.

110. Ashmanskas W, LeGeyt B, Newcomer F, Panetta J, Ryan W, Van Berg R, et al. Waveform-sampling electronics for a whole-body time-of-flight PET scanner. IEEE Trans Nucl Sci. 2014;61:1174-81.

111. Vinke R, Löhner H, Schaart D, Van Dam H, Seifert S, Beekman F, et al. Optimizing the timing resolution of SiPM sensors for use in TOF-PET detectors. Nucl Instrum Methods PhysRes, Sect A. 2009;610:188-91.

112. Szczęśniak T, Moszyński M, Nassalski A, Lavoute P, Dehaine A. A further study of timing with LSO on XP20D0 for TOF PET. IEEE Trans Nucl Sci. 2007;54:1464-73.

113. Bousselham AK. FPGA based data acquistion and digital pulse processing for PET and SPECT. 2007.

114. Wu J, Shi Z, Wang IY. Firmware-only implementation of time-todigital converter (TDC) in field-programmable gate array (FPGA). Nuclear Science Symposium Conference Record, 2003 IEEE. IEEE; 2003. p. 177-81.

115. Junnarkar SS, O'Connor P, Fontaine R. FPGA based self calibrating 40 picosecond resolution, wide range Time to Digital Converter. Nuclear Science Symposium Conference Record, 2008 NSS'08 IEEE. IEEE; 2008. p. 3434-9.

116. Hong KJ, Kim E, Yeom JY, Olcott P, Levin CS. FPGA-based time-to-digital converter for time-of-flight PET detector. Nuclear Science Symposium and Medical Imaging Conference (NSS/ MIC), 2012 IEEE. IEEE; 2012. p. 2463-5.

117. Wu J, Shi Z. The 10-ps wave union TDC: Improving FPGA TDC resolution beyond its cell delay. Nuclear Science Symposium Conference Record, 2008 NSS'08 IEEE: IEEE; 2008; p. 3440-6.

118. Fishburn MW, Menninga LH, Favi C, Charbon E. A 19.6 ps, FPGA-based TDC with multiple channels for open source applications. IEEE Trans Nucl Sci. 2013;60:2203-8.

119. Bayer E, Traxler M. A high-resolution (RMS) 48-channel time-todigital converter (TDC) implemented in a field programmable gate array (FPGA). IEEE Trans Nucl Sci. 2011;58:1547-52.

120. Szplet R, Jachna Z, Kwiatkowski P, Rozyc K. A 2.9 ps equivalent resolution interpolating time counter based on multiple independent coding lines. Meas Sci Technol. 2013;24, 035904.

121. Won JY, Kwon SI, Yoon HS, Ko GB, Son J-W, Lee JS. Dual-Phase Tapped-Delay-Line Time-to-Digital Converter With On-the-Fly Calibration Implemented in $40 \mathrm{~nm}$ FPGA. 2015. Biomedical Circuits and Systems, IEEE Transactions. IEEE Circuits and Systems Society. 2015. doi:10.1109/TBCAS.2015.2389227.

122. Kim E, Hong KJ, Yeom JY, Olcott PD, Levin CS. Trends of data path topologies for data acquisition systems in positron emission tomography. IEEE Trans Nucl Sci. 2013;60:3746-57. 